\title{
Channel Measurements for Free Space Optical Communication
}

\author{
Cristhof J. R. Runge, Maxwel V. da Silva and Jaime Portugheis
}

\begin{abstract}
This paper presents and analyzes fading measurements of the optical signal in a terrestrial free space optical communication link using a commercial system. Unlike previous works, the link distance is very short (about 90 meters). Nevertheless, it was possible to observe significant variations in the scintillation index over the course of one day. This behavior agrees with previous results obtained in the literature for much longer links.
\end{abstract}

Index Terms-Free space optical communications, channel measurements, scintillation index, fading.

\section{INTRODUCTION}

Current communication systems normally use one of two types of media for transmitting information: confined media, such as optical fibers and coaxial cables, and free space. In the case of free space transmission, radio frequency (range from $3 \mathrm{kHz}$ up to $60 \mathrm{GHz}$ ) and light (with wavelengths of $400 \mathrm{~nm}$ to $1550 \mathrm{~nm}$ ) are commonly used. Systems that use free space and light are referred to as OWC (Optical Wireless Communications) [1]. Among these OWC systems, those that are outdoors and terrestrial, are commonly known as FSO (Free Space Optics) systems. This paper considers FSO systems. These systems exhibit many advantages over traditional radio frequency (RF) systems, such as extensive range of free electromagnetic spectrum for use, highest rates in the order of Gbits/s, high immunity to electromagnetic interference, and also a low cost, when compared to systems using optical fibers or RF transmitters/receivers. However, optical propagation occurs through atmosphere and effects of absorption, scattering and turbulence are present and cause fading of the received signal in the receiver. Therefore, the design of an FSO system requires modeling the channel probability density function (pdf) in order to predict the received signal strength. These models are generally dependent on local climatic conditions. Even under climatic conditions of a clear sky, pressure differences and temperature variations caused by solar heating and wind change the air refractive index resulting in atmospheric turbulence. This turbulence causes variations in amplitude and phase of the received signal yielding fading phenomena [1]. Many experimental measures were performed to obtain a model pdf for this fading phenomena [2] - [6]. In

The material in this paper was presented in part at the XXXIII Simpósio Brasileiro de Telecomunicações, 2015.This work was supported in part by The State of São Paulo Research Foundation (FAPESP) under contract 2013/25977-7. C. J. R. Runge and J. Portugheis are with School of Technology, State University of Campinas, 13.484-332, Limeira, SP, Brazil, emails: <cristjrr,jaime>@ft.unicamp.br. M. V. da Silva is with Faculty of Anhanguera, 13482-231, Limeira, SP, Brazil, email: maxplanck07@hotmail.com

Digital Object Identifier:10.14209/jcis.2019.19 these measures, the link distances vary from $600 \mathrm{~m}$ up to 12 $\mathrm{km}$.

This paper describes measurement results of the intensity variation of the optical received signal on a terrestrial FSO link and analyzes the resulting fading. The link used to obtain these measurements is a commercial system and was installed on the roofs of two buildings in the campus of the School of Technology (State University of Campinas) in Limeira, São Paulo, Brazil. Unlike previous works, the link distance is about 90 meters, and the light beam passes close to the roofs of buildings in almost all its path. This is a typical connectivity scenario in enterprise/campus environments [1]. The measurements obtained show that even though it is a very short link, it is possible to observe the phenomenon of atmospheric turbulence.

\section{EXPERIMENTAL LINK}

The experimental link consists of a pair of TS802/ST transceivers of Optical Access Inc. Each transceiver consists of only one transmitter and one receiver. The light source of the transmitter consists of a laser operating at a wavelength of $850 \mathrm{~nm}$ with output power of $1 \mathrm{~mW}$ and divergence angle of $3 \mathrm{mrad}$. The receiver consists of a $\mathrm{Si}$ PIN photodiode with a field of view of $14 \mathrm{mrad}$, and the data interface uses an optical multimode fiber connection. For the experiment the transceiver was disassembled and separated into modules. After a detailed analysis of the internal circuits of equipment modules, it was possible to determine the monitoring point of the output voltage of the photodetector in order to collect measures corresponding to the received signal intensity. For the measurements we used an oscilloscope Tektronix TDS1000CEDU with digital storage. The sample rate was set to $25 \mathrm{kHz}$. The location of transceivers for the FSO link is a relevant question because it is necessary to ensure a good line of sight between the transceivers. They need to be immune to obstructions in the path of the optical signal, such as trees, leaves and birds [4], [7]. The trepidation is another critical item from the point of view of beam alignment. It is essential in the implementation of an FSO link the choice of fixing surfaces that should not be moved or subjected to trepidation [4]. Figure 1 shows the link location obtained using Google Earth software [8]. Both transceivers are located 5 meters from the ground. However, as shown in Figure 1, the optical signal is being transmitted through rooftops and close to them. Figure 2 shows the link transceiver used as receiver. As can be seen in Figure 2, there is a close proximity to the roof (only 28 centimeters). 


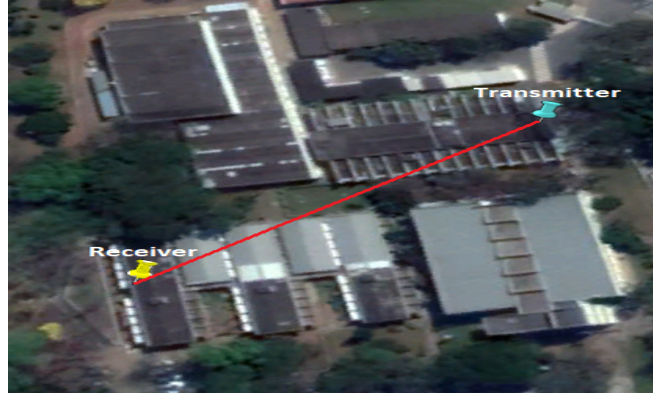

Fig. 1. Aerial view of the FSO link showing the location of the transmitter and receiver on the campus of the Faculty of Technology.

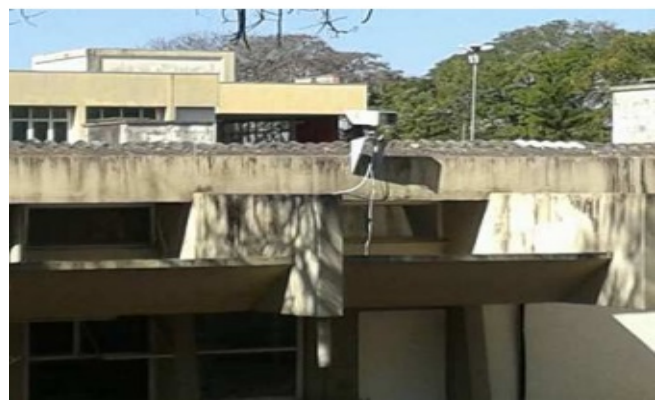

Fig. 2. View of the receiver installed on the roof of a building.

\section{Alignment Adjustments}

A very important aspect in a FSO link installation, consists of knowing the environmental conditions (rain, fog, drizzle or maritime proximity) under which the link will operate. These conditions can influence not only the link alignment but also the channel pdf statistics. In order to characterize the environmental condition of the link under investigation, a series of measurements were taken. Table I shows data collected in the month of August at the campus in Limeira, using the CPTEC/Limeira database that is available on the School page [9]. It shows values of Time, Weather (W), Temperature (T), Pressure (P), Visibility (V) and Wind Speed (WS).

Alignment adjustments between transceivers are one of the practical difficulties encountered in the initial phase of implementing an FSO link. In many situations, the link may experience a misalignment due to mechanical trepidation or even due to mechanical accommodation over time. Using an unmodulated continuos-wave $(\mathrm{CW})$ during the period shown in Table I it was possible to estimate the received signal intensity by measuring the $\mathrm{DC}$ value at the receiver. Figure 3 shows the temporal behavior of the DC voltage level at the receiver. These values serve as reference for the link operation and also indirectly provide the information about the misalignment of the link along the observed period of time. It can be seen that the average DC level decreases with time. Based on the results of Table I it is not possible to state that there is a correlation between this behavior and the weather conditions. However, mechanical accommodation (due to building sway) is likely to occur causing misalignment [1].
TABLE I

Climatic Conditions in Limeira (August)

\begin{tabular}{ccccccc}
\hline Day & Time & $\mathrm{W}$ & $\begin{array}{c}\mathrm{T} \\
\left({ }^{\circ} \mathrm{F}\right)\end{array}$ & $\begin{array}{c}\mathrm{P} \\
(\mathrm{kPa})\end{array}$ & $\begin{array}{c}\mathrm{V} \\
(\mathrm{km})\end{array}$ & $\begin{array}{c}\mathrm{WS} \\
(\mathrm{km} / \mathrm{h})\end{array}$ \\
\hline \multirow{2}{*}{ 1st } & $19: 15$ & clear & $78.8^{\circ}$ & 101.7 & 16 & 2 \\
& $06: 30$ & light mist & $55,4^{\circ}$ & 101.8 & 16 & 3 \\
& $13: 00$ & clear & $75.2^{\circ}$ & 102.0 & 16 & 6 \\
\hline \multirow{2}{*}{ 2nd } & $19: 00$ & rainy & $57.2^{\circ}$ & 102.0 & 8 & 14 \\
& $06: 00$ & clear & $57.2^{\circ}$ & 102.5 & 11 & 5 \\
& $13: 00$ & clear & $75.2^{\circ}$ & 102.8 & 16 & 6 \\
\hline \multirow{2}{*}{3 rd } & $18: 30$ & clear & $69.8^{\circ}$ & 102.0 & 14 & 8 \\
& $06: 00$ & cloudy & $59.0^{\circ}$ & 102.2 & 16 & 2 \\
& $13: 00$ & clear & $78.8^{\circ}$ & 102.1 & 16 & 3 \\
\hline \multirow{2}{*}{ 4th } & $19: 00$ & clear & $69.8^{\circ}$ & 102.1 & 16 & 7 \\
& $06: 15$ & clear & $55.4^{\circ}$ & 102.1 & 16 & 6 \\
& $13: 00$ & clear & $78.8^{\circ}$ & 102.0 & 16 & 6 \\
\hline \multirow{2}{*}{ 5th } & $19: 00$ & clear & $73.4^{\circ}$ & 102.1 & 16 & 6 \\
& $06: 00$ & light mist & $57.2^{\circ}$ & 102.2 & 16 & 4 \\
\hline \multirow{2}{*}{} & & & & & & \\
\hline
\end{tabular}

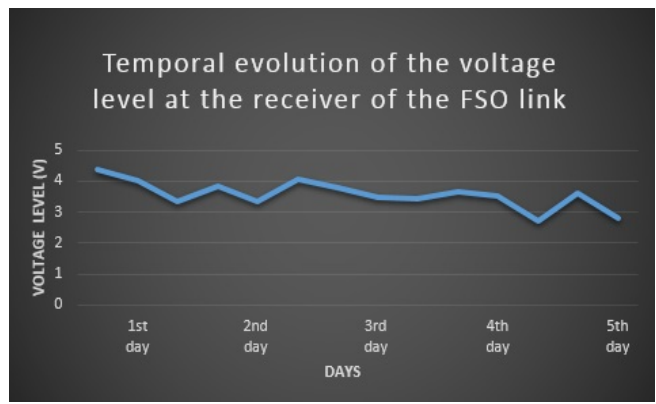

Fig. 3. Temporal behavior of the DC voltage level.

\section{Channel Model}

Consider a discrete time model for the samples collected by the oscilloscope given by the following expression [2],

$$
r_{k}=h_{k} x_{k}+n_{k},
$$

where the intensity $h_{k}$ (or channel gain) represents the fading due to atmospheric turbulence and $x_{k}$ the transmitted signal. An unmodulated $\mathrm{CW}$ is transmitted to measure the fading. This implies that neither the impulse response of the transmitter nor the receiver is considered in the model. Without loss of generality assume $x_{k}=1$. Also consider that the channel gain includes the responsiveness of the photodetector, the amplifier gain at the receiver, propagation losses, atmospheric losses and fading due to the scintillation phenomenon [6].

As already shown in previous experimental works [3], [6], the combined effect of thermal noise at the receiver and the background radiation noise can be modeled as an additive noise denoted by $n_{k}$ in Equation 1. In [3] a long experimental link $(12 \mathrm{~km})$ working at a low signal-to-noise ratio (SNR) was analyzed. The additive noise was not neglected and the focus of the work was to estimate the pdf of the intensity $h_{k}$ with prior knowledge of the mean and variance of the additive noise. It is shown in [3] that if the variance of the additive noise is of the order of the logarithm of the intensity variance (SNR low), the noise can not be neglected. But if the variance of the additive noise is much smaller (at least 10 times) of the logarithm of the intensity variance (high SNR), the noise can be neglected. Measurements of the received signal, $r_{k}$, were carried out for an experimental $1 \mathrm{~km}$ link and are described 
in [5]. These measures were used to estimate the variance of the intensity, $h_{k}$, where the effect of the additive noise was neglected (this assumes that $r_{k}=h_{k}$ ). In [6] the samples of the received signal in a $1.87 \mathrm{~km}$ link were collected and processed using a notch filter to minimize the effect of the additive noise.

\section{Channel Measurements And Analysis}

In this section measurement results are presented and channel pdfs modeling channel gain and additive noise are given. These channel functions were obtained using a fitting program. In the presented graphics the histogram of the collected measurements and the resulted fitting functions are compared. The behavior of the received signal in time domain is also showed. As in Section III, all measurements were obtained by setting the transmitter to an unmodulatd $\mathrm{CW}$ mode.

\section{A. Scintillation Index}

The normalized variance of the channel gain, $h$, is called scintillation index of the FSO channel and is given by [10]

$$
\sigma_{I}^{2}=\frac{E\left[h^{2}\right]-E[h]^{2}}{E[h]^{2}}
$$

where $E[\cdot]$ denotes expected value. If $E[h]=1, \sigma_{I}^{2}$ is equal to variance of $h$, and hence can be viewed as the scintillation index [11]. The scintillation index can be estimated from samples collected by the oscilloscope using the expressions:

$$
E\left[h^{2}\right]-E[h]^{2}=\frac{1}{N} \sum_{k=1}^{N}\left(h_{k}-E[h]\right)^{2}
$$

and

$$
E[h]=\frac{1}{N} \sum_{k=1}^{N} h_{k}
$$

where $N$ is the number of samples collected.

Depending on the value of $\sigma_{I}^{2}$, three distinct regimes of turbulence may be defined [2]: weak turbulence $\left(\sigma_{I}^{2}<1 / 2\right)$, moderate turbulence $\left(1 / 2<\sigma_{I}^{2}<1\right)$ and strong turbulence $\left(\sigma_{I}^{2}>1\right)$. For weak turbulence, the channel gains are modeled with a lognormal pdf:

$$
f(h)=\frac{1}{h \sqrt{2 \pi \sigma^{2}}} \exp \left[-\frac{\left(\ln (h)+\sigma^{2} / 2\right)^{2}}{2 \sigma^{2}}\right]
$$

where $E[h]=1$ and $\sigma^{2}=\ln \left(\sigma_{I}^{2}+1\right)$.

For weak to moderate turbulence, the most suitable mathematical function to model channel gains is a gamma-gamma density function [12]. And for strong turbulence, a model of negative exponential is usually chosen [2]. Due to the fact that the paper considers a short link, it is expected to observe only weak or moderate turbulence. In order to estimate scintillation indexes, a series of measurements was carried out in August. Table II shows the estimated scintillation values. The measurements shown in lines "Morning", "Afternoon" and "Night" were collected around 6:00 o'clock, 13:00 o'clock and 19:00 o'clock, respectively. The letters T, W and C refer to Temperature (in degrees Fahrenheit), Wind (in $\mathrm{km} / \mathrm{h}$ ) and Climate, respectively. Analyzing the data in Table II, one can try to investigate the cross-correlation of $\mathrm{T}, \mathrm{W}$, and $\mathrm{C}$ with the estimated values of scintillation indices. This attempt was made in [5] and [6]. However, this paper confines itself to the following conclusion [3]: the highest scintillation indexes are observed close to noon (13:00 o'clock). It is also worth emphasizing that in column "Measure 2" of Table II the scintillation index in the afternoon is approximately 10 times greater than that in the morning.

TABLE II

ESTIMATED VALUES OF SCINTILLATION INDEX

\begin{tabular}{|c||c|c|c|}
\hline & MEASURE 1 & MEASURE 2 & MEASURE 3 \\
\hline \hline Morning & $\mathbf{0 . 0 0 0 0 1 4 7}$ & $\mathbf{0 . 0 0 0 0 2 5 2}$ & $\mathbf{0 . 0 0 0 0 2 0 2}$ \\
\hline T & 66.92 & 61.52 & 58.28 \\
\hline W & 12.2 & 14.3 & 12.6 \\
\hline C & cloudy & Clear & cloudy \\
\hline Afternoon & $\mathbf{0 . 0 0 0 0 5 8 3}$ & $\mathbf{0 . 0 0 0 2 3 8 3}$ & $\mathbf{0 . 0 0 0 2 1 1 5}$ \\
\hline T & 69.8 & 77.18 & 77.9 \\
\hline W & 15.7 & 15.1 & 12.8 \\
\hline C & clear & clear & clear \\
\hline Night & $\mathbf{0 . 0 0 0 2 6 7}$ & $\mathbf{0 . 0 0 0 0 2 9 4}$ & $\mathbf{0 . 0 0 0 0 3 1 5}$ \\
\hline T & 80.24 & 57.56 & 74,66 \\
\hline W & 12.8 & 12.1 & 15.8 \\
\hline C & clear & rainy & clear \\
\hline \multicolumn{4}{|r}{} \\
\end{tabular}

\section{B. Noise}

The additive noise described in Equation 1 is modeled as the sum of the thermal and background radiation noise at the receiver. To obtain the corresponding parameters, first the transmitter is turned off, and then samples of the oscilloscope are collected and measured. Then the receiver is covered by a dark board and now samples due to only thermal noise are collected and measured. Figure 4 shows both the histogram and the Gaussian pdf obtained using a program to fit a normal distribution to the data collected.

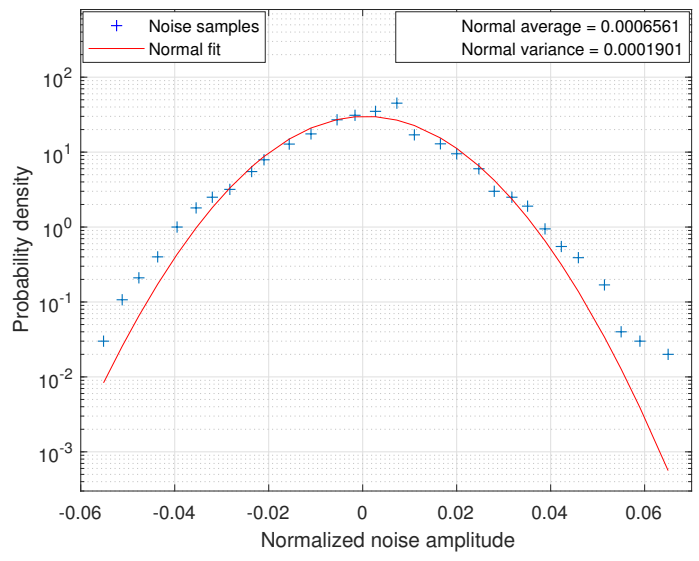

Fig. 4. Histogram of thermal noise and adjusted Gaussian density.

As in [3] and [6], no significant difference was observed between statistics of total additive noise and only-thermal additive noise. Figure 4 corresponds to the result obtained using the fitting function normfit of Matlab software for 24.820 samples collected by the oscilloscope. A good agreement of the fitted function to the data can be observed.

\section{Channel Gain}

Figure 5 shows a 1 second segment of a detected optical signal distorted by atmospheric turbulence with a scintillation 


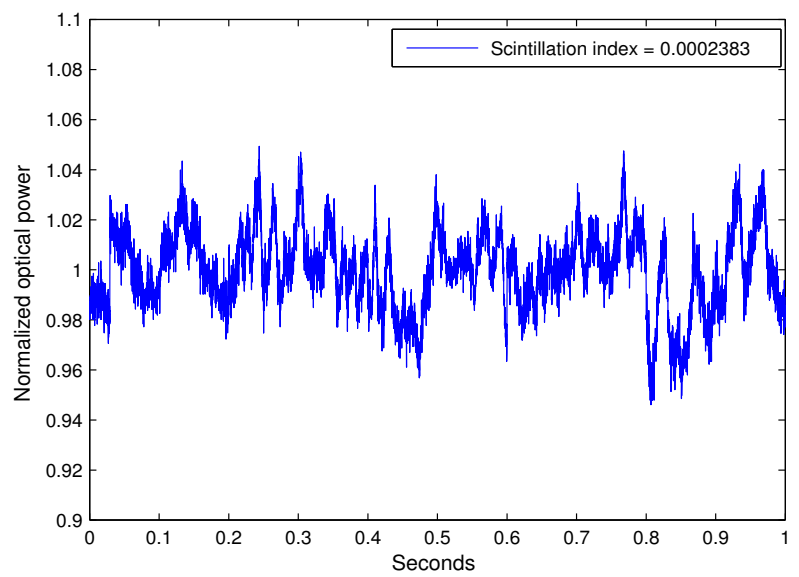

Fig. 5. Waveform of normalized channel gain for a scintillation index value of 0.0002383 (one second segment).

index of 0.0002383. The signal was recorded around 13:00 o'clock under clear sky condition. Optical power is normalized by the average intensity received.

Figure 6 shows both the histogram and the lognormal (LN) pdf obtained for the same day and time of Figure 5. In this case the Matlab lognfit function, which is written based on the maximum likelihood method, was used. A total of 12.410 samples were collected by the oscilloscope. A good agreement of the fitted function to the data is also observed.

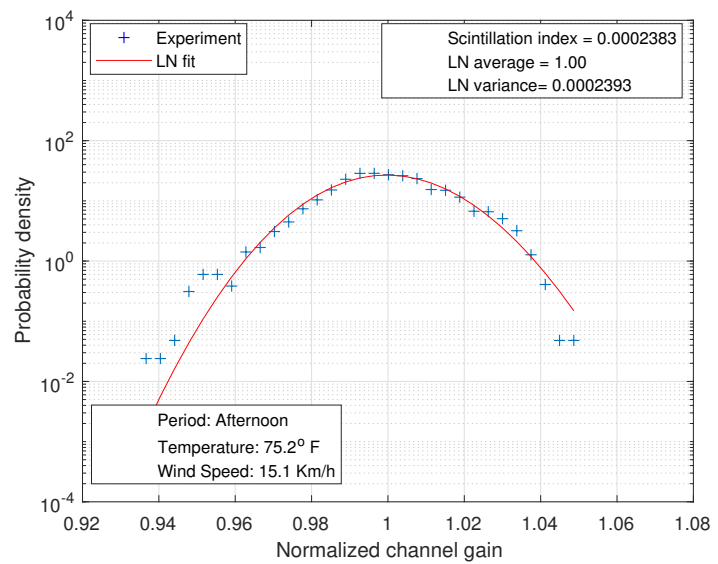

Fig. 6. Histogram of the normalized channel gain and the LN density adjusted for an afternoon period and clear sky climatic conditions. Expected value of $h, E[h]=3.36 \mathrm{~V}$.

Figure 7 shows the histogram and the fitted LN density for data collected around 6:00 o'clock whereas Figure 8 shows for data collected around 19:00 o'clock. The results in both figures were also generated with a total of 12.410 samples.

It is worth emphasizing that comparing the variance of the additive noise with the logarithm of the estimated intensity variance, for all cases, the first is at least 25 times smaller than the others. In other words, one has a high SNR situation. Thus, for adjustments shown in Figures 6, 7 and 8, the additive noise can be neglected.

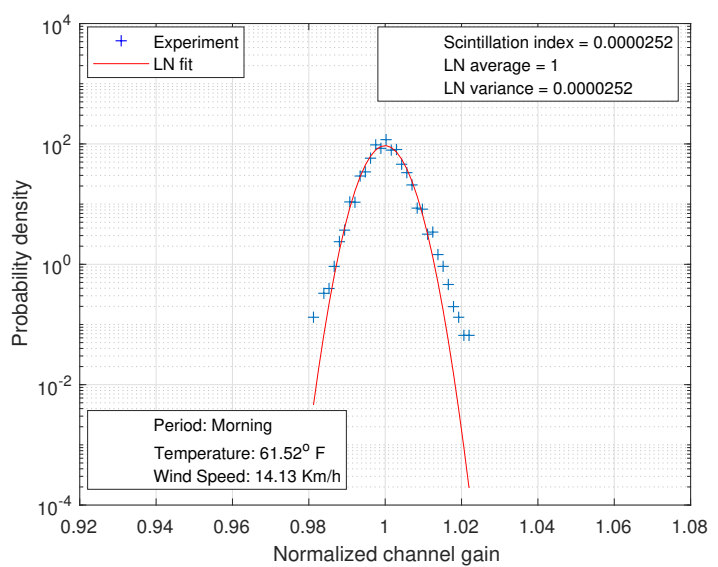

Fig. 7. Histogram of normalized channel gain and the LN density adjusted for a morning period and clear sky climatic conditions. Expected value of $h$, $E[h]=3.78 \mathrm{~V}$.

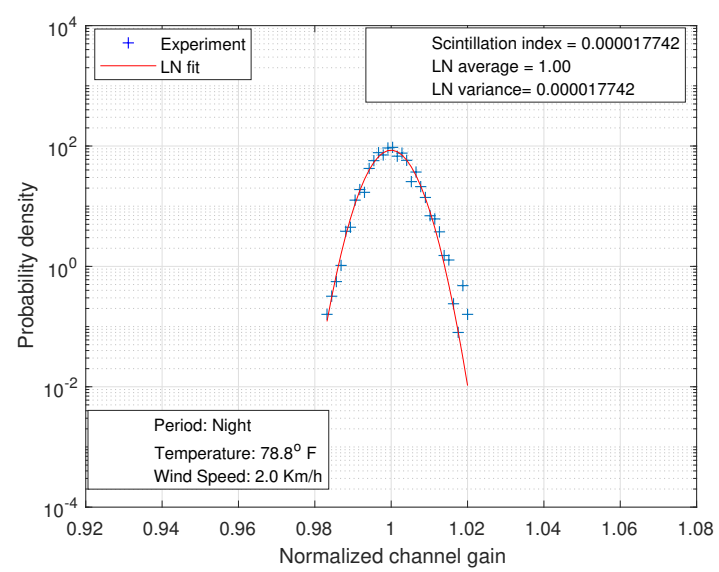

Fig. 8. Histogram of normalized channel gain and the LN density adjusted for a night period and clear sky climatic conditions. Expected value of $h$, $E[h]=4.4 \mathrm{~V}$.

Another interesting fact is that the highest scintillation index is observed in the afternoon (Figure 6), in agreement with what was discussed in Section V. Some of the observed low scintillation index values are close to those obtained in [5] for a link with a distance of $1 \mathrm{~km}$, that is, more than 10 times longer than the one used in this work. The authors in [5] showed that these low values are related to local weather conditions like temperature and rain fall. Based on the results of [5], we believe that it is possible to justify the observed low scintillation values by both the relatively short length of the link and the fact that the measurements were performed in a local with typical tropical climate characteristics where extreme climatic weather conditions are rare.

\section{FINAL REMARK}

The scintillation indexes of Table II characterize a regime of weak turbulence and its channel gains were modeled here with a lognormal pdf. The gamma-gamma pdf is also suitable to fit with measurements under weak turbulence [12]. We intend to compare the results presented here with other obtained by fitting with the gamma-gamma pdf in future work. 


\section{REFERENCES}

[1] M. A. Khalighi and M. Uysal, "Survey on Free Space Optical Communication: A Communication Theory Perspective," IEEE Commun. Surveys Tuts., vol. 16, no. 4, pp. 2231-2258, June 2014, DOI:10.1109/COMST.2014.2329501.

[2] J. Anguita, M. Neifeld, B. Hildner and B. Vasic, "Rateless Coding on Experimental Temporally Correlated FSO Channels," J. of Lightw. Technol., vol. 28, no. 7, pp. 990-1002, Apr. 2010, DOI: $10.1109 /$ JLT.2010.2040136.

[3] A. Khatoon, W. G. Cowley and N. Letzepis, "Channel measurement and estimation for free space optical communications," Proc. of the AusCTW, pp. 112-117, Jan.-Feb. 2011, DOI:10.1109/AUSCTW.2011.5728747.

[4] F. S. Marzano, S. Mori, F. Frezza, P. Nocito, G. M. T. Beleffi, G. Incerti, E. Restuccia, and F. Consalvi, "Free-space optical high-speed link in the urban area of southern Rome: preliminary experimental set up and channel modelling," Proc. of the 5th EUCAP, pp. 2737-2741, Apr. 2011

[5] K. H. Kim, T. Higashino, K. Tsukamoto and S. Komaki, "Optical fading analysis considering spectrum of optical scintillation in terrestrial freespace optical channel," Proc. of the ICSOS, pp. 58-66, May 2011, DOI:10.1109/ICSOS.2011.5783710.

[6] A. Mostafa and S. Hranilovic, "Channel Measurement and Markov Modeling of an Urban Free-Space Optical Link," J. Opt. Commun. Netw., vol. 4, no. 10, pp. 836-846, Oct. 2012, DOI:10.1364/JOCN.4.000836.

[7] H. Henniger and O. Wilfert, "An Introduction to Free-space Optical Communications," Radioengineering, vol. 19, no. 2, pp. 203-212, 2010.

[8] http://www.google.com.br/intl/pt-BR/earth/learn/

[9] Page of the School of Technology in the Internet CPTEC/LIMEIRA: http://www.ft.unicamp.br/dadosmeteorologicos.

[10] L. Zhang, Z. Wu, Y. Zhang and H. Detian, "Improved maximum likelihood detection for mitigating fading estimation error in free space optical communication," Optical Engineering, vol. 52(1), 015004, pp. 1-6, Jan. 2013,DOI:10.1117/1.OE.52.1.015004.

[11] K. Kiasaleh, "Channel estimation for FSO channels subject to GammaGamma turbulence," Proc. of the ICSOS, pp. 1-7, Oct. 2012

[12] Z. Ghassemlooy, W. Popoola and S. Rajbhandari, Optical Wireless Communications: System and Channel Modelling with MATLAB , CRC Press, 2012.

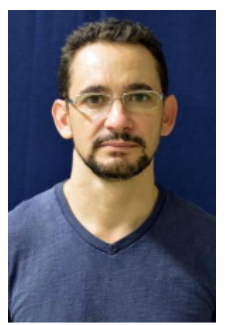

Cristhof Johann Roosen Runge received a degree in Electrical Engineering from the Federal University of Santa Catarina (1995), a Master's degree in Electrical Engineering from the State University of Campinas (1997) and a PhD in Electrical Engineering from the University of São Paulo (2012). He worked for 15 years in the telecommunications industry in the area of equipment development. He is currently Assistent Professor at the School of Technology of the State University of Campinas. He is interested in digital wireless RF and optical transmission systems, error correction codes, digital modulation and demodulation and signal processing, mobile devices, IoTs and machine learning.

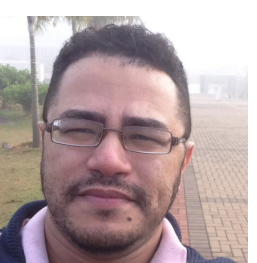

Maxwel Vitorino da Silva received a degree in Communication System Technology, in Communication Engineering and a Master's degree in Technology from the State University of Campinas in 2014, 2017 and 2017, respectively. He is now with Faculty of Anhanguera, Limeira, where he teaches courses and acts as coordinator of the Control and Automation Engineering and Computer Science Courses. His research interests are in the areas of Digital Transmission and Applied Coding and Information Theory.

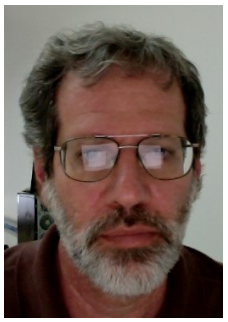

Jaime Portugheis received a degree in Electrical Engineering from the Federal University of Pernambuco (1983), a Master's degree in Electrical Engineering from the State University of Campinas (1987) and a $\mathrm{PhD}$ in Electrical Engineering from Darmstadt University of Technology, Germany (1992). He is currently a Full Professor at the School of Technology of the State University of Campinas. $\mathrm{He}$ is Senior Member of the Brazilian Telecommunications Society. His research interests are in the areas of Digital Transmission, Information Theory and Applied Coding, with emphasis on algorithms for iterative receivers, coded modulation and error-correcting codes. 